\title{
Effect of $\beta$-blockers on the risk of COPD exacerbations according to indication of use: the Rotterdam Study
}

\author{
Leila Karimi $\mathbb{1}^{1}$, Lies Lahousse $\mathbb{1}^{2,3}$, Phebe De Nocker ${ }^{2}$, Bruno H. Stricker ${ }^{3,4}$, \\ Guy G. Brusselle $\mathbb{1}^{3,5,6}$ and Katia M.C. Verhamme (1) ${ }^{1,2}$
}

Affiliations: ${ }^{1}$ Dept of Medical Informatics, Erasmus University Medical Center, Rotterdam, The Netherlands. ${ }^{2}$ Dept of Bioanalysis, Ghent University, Ghent, Belgium. ${ }^{3}$ Dept of Epidemiology, Erasmus University Medical Center, Rotterdam, The Netherlands. ${ }^{4}$ Dept of Internal Medicine, Erasmus University Medical Center, Rotterdam, The Netherlands. ${ }^{5}$ Dept of Respiratory Medicine, Ghent University Hospital, Ghent, Belgium. ${ }^{6}$ Dept of Respiratory Medicine, Erasmus University Medical Center, Rotterdam, The Netherlands.

Correspondence: Bruno H. Stricker, Dept of Epidemiology, Erasmus MC, Postbus 2040, 3000 CA Rotterdam, Netherlands. E-mail: b.strickerderasmusmc.nl

ABSTRACT Observational studies report a reduction of COPD exacerbations in patients treated with $\beta$ blockers. In contrast, the Beta-Blockers for the Prevention of Acute Exacerbations of Chronic Obstructive Pulmonary Disease (BLOCK COPD) randomised controlled trial which excluded COPD patients with cardiovascular conditions showed an increase in COPD exacerbations. It is unclear whether this discrepancy could be explained by underlying cardiovascular comorbidity. We examined whether the association between use of $\beta$-blockers and risk of COPD exacerbations differed between patients with and without a cardiovascular indication for $\beta$-blockers use.

Within the Rotterdam Study, we followed COPD subjects until the first COPD exacerbation, or end of follow-up. Cardiovascular indication for $\beta$-blockers use was defined as a history of hypertension, coronary heart disease, atrial fibrillation and/or heart failure at baseline. The association between $\beta$-blockers use and COPD exacerbations was assessed using Cox proportional hazards models adjusted for age, sex, smoking, incident cardiovascular disease (i.e. heart failure, hypertension, atrial fibrillation and/or coronary heart disease during follow-up), respiratory drugs and nitrates.

In total, 1312 COPD patients with a mean age of $69.7 \pm 9.2$ years were included. In patients with a cardiovascular indication ( $\mathrm{n}=755$, mean age of $70.4 \pm 8.8$ years), current use of cardioselective $\beta$-blockers was significantly associated with a reduced risk of COPD exacerbations (HR 0.69, 95\% CI 0.57-0.85). In contrast, in subjects without a cardiovascular indication $(n=557$, mean age of $68.8 \pm 9.7$ years), current use of cardioselective $\beta$-blockers was not associated with an altered risk of COPD exacerbations (HR 0.94, 95\% CI 0.55-1.62).

Use of cardioselective $\beta$-blockers reduced the risk of exacerbations in COPD patients with concomitant cardiovascular disease. Therefore, the potential benefits of $\beta$-blockers might be confined to COPD patients with cardiovascular disease.

@ERSpublications

Use of cardioselective $\beta$-blockers reduces the risk of COPD exacerbations in patients with concomitant cardiovascular disease. The potential benefits of $\beta$-blockers might be restricted to COPD patients with cardiovascular disease. https://bit.ly/3bB1RGg

Cite this article as: Karimi L, Lahousse L, De Nocker P, et al. Effect of $\beta$-blockers on the risk of COPD exacerbations according to indication of use: the Rotterdam Study. ERJ Open Res 2021; 7: 00624-2020 [https://doi.org/10.1183/23120541.00624-2020].

Received: 28 Aug 2020 | Accepted: 16 Feb 2021

Copyright $\odot$ The authors 2021. This version is distributed under the terms of the Creative Commons Attribution NonCommercial Licence 4.0. For commercial reproduction rights and permissions contact permissions@ersnet.org 


\section{Introduction}

Chronic obstructive pulmonary disease (COPD) is a leading cause of death worldwide [1, 2]. COPD exacerbations that are related to poor prognosis and severe COPD exacerbations requiring hospital admission increase the total costs due to COPD management [2,3]. Cardiovascular comorbidities encompassing arterial hypertension, ischaemic heart disease, atrial fibrillation and heart failure are common in patients with COPD [4]. Based on the recommendations of the The Global Initiative for Chronic Obstructive Lung Disease (GOLD) guidelines, comorbidities in COPD patients should be treated according to the standard strategies irrespective of the presence of COPD [2]. $\beta$-blockers are recommended for the first-line treatment of several cardiovascular conditions, including coronary artery disease, heart failure, atrial fibrillation and hypertension (in case of concomitant with heart failure, angina pectoris, or recent myocardial infarction) [5-7].

Observational studies have already investigated the beneficial effect of the use of $\beta$-blockers in patients with COPD, but not stratified by the presence of cardiovascular disease $[8,9]$. The effect of $\beta$-blockers use on survival and exacerbations has also been examined in patients with COPD and hypertension $[10,11]$. While observational studies and large meta-analysis reported that the use of $\beta$-blockers is associated with reductions in mortality, hospital admissions and exacerbations in COPD patients $[8,9,11-15]$, physicians are still reluctant to prescribe $\beta$-blockers in patients with cardiovascular disease and concomitant COPD due to concerns of the potential risk of $\beta$-blockers induced bronchoconstriction [16-18]. Recently, the Beta-Blockers for the Prevention of Acute Exacerbations of Chronic Obstructive Pulmonary Disease (BLOCK COPD) trial, a double-blind placebo-controlled randomised clinical trial (RCT), reported an increased risk of severe (leading to hospitalisation), and very severe (leading to intubation and mechanical ventilation) COPD exacerbations in metoprolol treated COPD patients without an indication for the use of $\beta$-blockers. However, the time until the first COPD exacerbation was similar in the metoprolol group and the placebo group [19]. It is still unclear whether the current discrepancy between findings from observational studies and the BLOCK COPD RCT could be explained by underlying cardiovascular comorbidity.

Therefore, we aimed to examine whether the association between the use of $\beta$-blockers and the risk of COPD exacerbations differed between patients with and without a cardiovascular indication for the use of $\beta$-blockers.

\section{Methods}

\section{Setting and study population}

The present study was performed within the Rotterdam Study, an ongoing prospective population-based cohort study in the well-defined Ommoord district in the city of Rotterdam in the Netherlands [20] The Rotterdam Study comprises approximately 15000 participants, aged $\geqslant 45$ years, and includes four sub-cohorts (RS-I, RS-II, RS-III and RS-IV). Baseline data were collected from 1989 to 1992 in RS-I ( $n=7983)$, from 2000 to 2003 in RS-II ( $\mathrm{n}=3011)$, from 2006 to 2009 in RS-III ( $\mathrm{n}=3932$ ), and RS-IV $(n=4000)$ was established in 2016. Follow-up examinations were conducted periodically, which consisted of a home interview and an extensive set of tests at the research facility. In addition, the relevant data were retrieved from the medical records of the general practitioners, nursing homes and hospitals. The Medical Ethics Committee of the Erasmus Medical Center approved the Rotterdam Study. All participants provided written informed consent.

The study population for the analyses consisted of COPD patients who gave informed consent for follow-up monitoring and had pharmacy and covariables data available until January 1, 2011.

\section{COPD and COPD exacerbations}

The diagnosis of COPD was confirmed by pre-bronchodilator obstructive spirometry (forced expiratory volume in $1 \mathrm{~s}\left(\mathrm{FEV}_{1}\right) /$ forced vital capacity $\left.(\mathrm{FVC})<0.7\right)$. If an interpretable spirometry was not available in the Rotterdam Study, the use of respiratory drugs (Anatomical Therapeutic Chemical Classification codes: R03) was exclusively used for potential case finding; each potential case was validated through evaluation of all medical records, specialist letters and hospitalisation. COPD cases were then identified as having a clear and well-founded physician diagnosis of COPD base on clinical presentation and/or lung function assessed by the general practitioner or respiratory physician [21]. Prevalent COPD was defined as COPD diagnosed before the study start, and incident COPD was defined as the first diagnosis of COPD during follow-up. The start date of follow-up was defined as the date of study enrolment for prevalent COPD or the date of diagnosis for incident COPD. We followed COPD patients until the first COPD exacerbation, death, lost to follow-up or the end of the follow-up (i.e. January 1, 2011), whichever came first. COPD exacerbations were defined as acute episodes of worsening of respiratory symptoms requiring use of either systemic corticosteroids and/or antibiotics (moderate exacerbations), or requiring hospitalisation (severe 
exacerbations) $[22,23]$. The outcome was defined as the first moderate to severe COPD exacerbation during follow-up and the date of outcome was taken as the index date.

\section{Drug exposure}

We obtained medication dispensing data from the computerised pharmacies in the study district. Records of all filled prescriptions from January 1, 1991 onwards were available and included information on the product name, the Anatomical Therapeutic Chemical Classification codes, the dispensing date, the prescribed dosing regimen, and the amount dispensed. The exposure of interest was $\beta$-blockers (C07) and these were categorised into non-cardioselective $\beta$-blockers (C07AA) and cardioselective $\beta$-blockers $(\mathrm{C} 07 \mathrm{AB})$. Patients were considered as "current users" if they used a $\beta$-blocker on the day of the first exacerbation (i.e. the index date) or when the last day of use of $\beta$-blockers fell within 30 days prior to the index date. If the last day of use of $\beta$-blockers was more than 30 days prior to the index date, subjects were considered as "past users". Patients were considered as "non-users", if they did not use $\beta$-blockers prior to the first exacerbation during the study period.

\section{Co-variables}

Several co-variables were considered as potential confounding factors such as: age at index date, sex, smoking, body mass index (BMI), use of respiratory drugs (R03), and use of cardiovascular drugs (i.e., diuretics (C03), calcium antagonists (C08), agents acting on the renin-angiotensin system (C09), antiarrhythmics (C01), nitrates (C01DA), and lipid-lowering drugs (C10)). Incident cardiovascular disease (i.e. arterial hypertension, coronary heart disease, atrial fibrillation and/or heart failure during follow-up), as a time-varying determinant, was also considered as a confounder. BMI was calculated as weight divided by height squared $\left(\mathrm{kg} \cdot \mathrm{m}^{-2}\right)$. Hypertension was defined as a resting blood pressure above $140 / 90 \mathrm{mmHg}$ or the use of blood pressure-lowering medication. The diagnosis of heart failure was during follow-up using the medical records of the participants [24]. Coronary heart disease (CHD) was defined as a compound outcome including fatal or nonfatal myocardial infarction or CHD mortality [24]. Data on smoking were obtained from questionnaires and were categorised into "never" or "ever-smokers".

\section{Statistical analyses}

Continuous variables were presented as mean with standard deviation (SD) and as median and interquartile range, where appropriate. Categorical variables were described as counts (n) and proportions (\%). Quantitative variables were statistically compared with a Student's t-test (parametric) or Wilcoxon signed rank sum test (non-parametric, when necessary). Categorical variables were statistically compared with Chi-squared test.

The use of $\beta$-blockers, other medications, age at index date and incident cardiovascular disease were included in the models as time-varying determinants [25]. Individuals were considered to have a cardiovascular indication for treatment with $\beta$-blockers in case they were diagnosed with arterial hypertension, coronary heart disease, atrial fibrillation and/or heart failure at baseline. In the main analysis, the total population was stratified into participants with or without a cardiovascular indication for the use of $\beta$-blockers at baseline.

The association between the use of $\beta$-blockers and COPD exacerbations was assessed using Cox proportional hazards models, adjusted for age, sex, smoking and other factors that changed the crude estimate for current use of $\beta$-blockers by more than $10 \%$ (i.e. incident cardiovascular disease during follow-up, use of respiratory drugs and nitrates). $\beta$-blockers are currently no longer considered as first-line treatment for hypertension in COPD [26]. Therefore, in a sensitivity analysis, the patients with a cardiovascular indication for the use of $\beta$-blockers were stratified into two strata: 1 ) only hypertension; and 2) coronary heart disease, atrial fibrillation and/or heart failure with or without hypertension (strict cardiovascular indication). Furthermore, to assess the effect of long-term use of $\beta$-blockers, we repeated the analysis for long term use of $\beta$-blockers defined as the use of $\beta$-blockers for at least 270 days during one year prior to the index date. A p-value $<0.05$ was considered statistically significant. All statistical analyses were conducted using the statistical software package SPSS/24.0 and package R (version 3.3.3).

\section{Results}

\section{COPD patients characteristics}

The study flow of participants is described in figure 1. The COPD patients characteristics are shown in table 1. A total of 1312 COPD patients with a mean \pm SD age of $69.7 \pm 9.2$ years were included. Males comprised $57.2 \%(n=750)$ of the cohort and $84.1 \%(n=1104)$ were ever smokers. The median (interquartile range) follow-up time was 426 (155-1037) days. At the end of follow-up, 1055 (80.4\%) first COPD exacerbations were recorded. Patients with a cardiovascular indication for the use of $\beta$-blockers $(n=755$, age $=70.4 \pm 8.8$ years, sex male $=58.0 \%)$ were significantly $(\mathrm{p}=0.002)$ older than those $(n=557$, 
FIGURE 1 Flowchart of participants. ACO: asthma-COPD overlap; COPD patients were included until January 1, 2011.

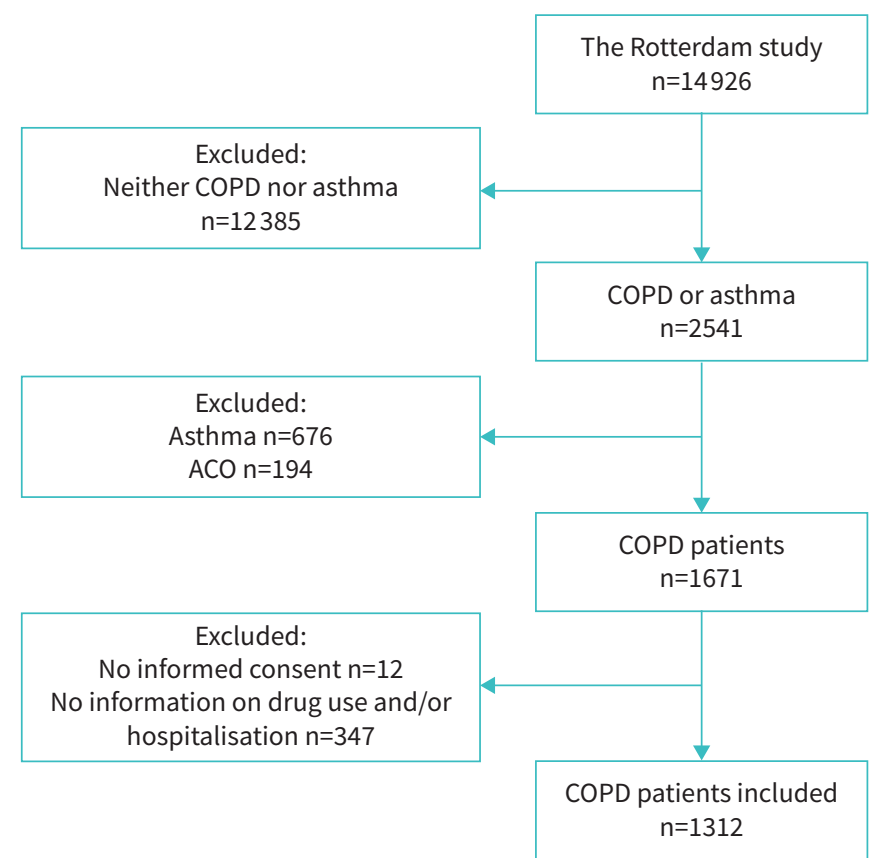

age $=68.8 \pm 9.7$ years. sex male $=56.0 \%$ ) without a cardiovascular indication for the use of $\beta$-blockers. During follow-up, several subjects developed cardiovascular events in both categories with and without a cardiovascular indication for the use of $\beta$-blockers (116 (15.4\%) and 128 (23.0\%), respectively).

\section{$\boldsymbol{\beta}$-blockers use and risk of COPD exacerbations}

In the total population, current use of cardioselective $\beta$-blockers, compared with non-use, was associated with a reduced risk of exacerbations (HR 0.69, 95\% CI 0.58-0.83). In patients with a cardiovascular indication for the use of $\beta$-blockers (table 2), current use of cardioselective $\beta$-blockers reduced the risk of COPD exacerbations by $31 \%$ (HR $0.69,95 \%$ CI 0.57-0.85). In contrast, in subjects without a cardiovascular indication for the use of $\beta$-blockers, current use of cardioselective $\beta$-blockers did not alter the risk of COPD exacerbations (HR 0.94, 95\% CI 0.55-1.62).

The results of sensitivity analysis showed that current use of cardioselective $\beta$-blockers significantly reduced the risk of COPD exacerbations across two strata of cardiovascular indication of $\beta$-blockers use, HR 0.64 (95\% CI 0.44-0.91) in subjects with a strict cardiovascular indication for the use $\beta$-blockers

\section{TABLE 1 Patients characteristics at baseline}

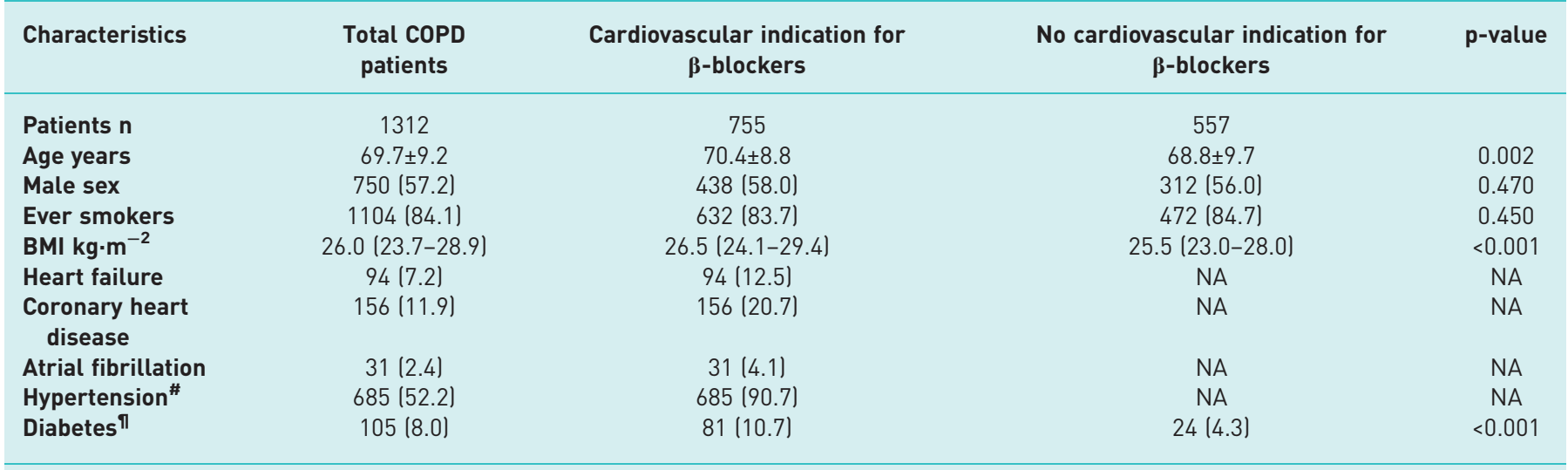

Data are presented as mean $\pm \mathrm{SD}, \mathrm{n}(\%)$ or median (interquartile range), unless otherwise stated. Cardiovascular indication for $\beta$-blockers use was defined as a history of hypertension, coronary heart disease, atrial fibrillation and/or heart failure at baseline. BMI: body mass index; NA: not applicable. \#: data were missing on hypertension in 218 subjects; ๆ: diabetes mellitus was defined as a fasting serum glucose concentration of $\geqslant 7.0 \mathrm{mmol} \cdot \mathrm{L}^{-1}$ or a non-fasting serum glucose concentration of $\geqslant 11.1 \mathrm{mmol} \cdot \mathrm{L}^{-1}$ or the use of blood glucose-lowering medications [40]. 
TABLE 2 Use of $\beta$-blockers and risk of COPD exacerbations

\begin{tabular}{|c|c|c|c|c|c|}
\hline$\beta$-blockers & Exacerbations $\mathbf{n}$ & Crude hazard ratio $(95 \% \mathrm{Cl})$ & p-value & Adjusted $^{\#}$ hazard ratio $(95 \% \mathrm{Cl})$ & p-value \\
\hline \multicolumn{6}{|c|}{ Total COPD (n=1312) } \\
\hline No use & 731 & Reference & & Reference & \\
\hline \multicolumn{6}{|c|}{ Cardioselective $\beta$-blockers } \\
\hline Current use & 161 & $0.74(0.62-0.88)$ & 0.001 & $0.69(0.58-0.83)$ & 0.00005 \\
\hline Past use & 95 & $1.02(0.83-1.27)$ & 0.822 & $1.01(0.81-1.26)$ & 0.915 \\
\hline \multicolumn{6}{|c|}{ Non-cardioselective $\boldsymbol{\beta}$-blockers } \\
\hline \multicolumn{6}{|c|}{ No cardiovascular indication for $\beta$-blockers use $(n=557)$} \\
\hline No use & 400 & Reference & & Reference & \\
\hline \multicolumn{6}{|c|}{ Cardioselective $\beta$-blockers } \\
\hline Current use & 18 & $1.38(0.85-2.23)$ & 0.193 & $0.94(0.55-1.62)$ & 0.835 \\
\hline Past use & 21 & $1.02(0.66-1.58)$ & 0.934 & $0.98(0.62-1.53)$ & 0.918 \\
\hline \multicolumn{6}{|c|}{ Non-cardioselective $\boldsymbol{\beta}$-blockers } \\
\hline \multicolumn{6}{|c|}{ Cardioselective $\boldsymbol{\beta}$-blockers } \\
\hline Current use & 143 & $0.71(0.58-0.86)$ & 0.001 & $0.69(0.57-0.85)$ & 0.0004 \\
\hline Past use & 74 & $1.03(0.80-1.33)$ & 0.788 & $1.08(0.83-1.40)$ & 0.560 \\
\hline \multicolumn{6}{|c|}{ Non-cardioselective $\boldsymbol{\beta}$-blockers } \\
\hline Current use & 23 & $1.15(0.75-1.75)$ & 0.528 & $1.38(0.89-2.12)$ & 0.147 \\
\hline Past use & 26 & $0.87(0.58-1.30)$ & 0.498 & $0.71(0.47-1.07)$ & 0.106 \\
\hline
\end{tabular}

(i.e., ischaemic heart disease, atrial fibrillation and/or heart failure) and HR 0.69 (95\% CI 0.54-0.89) in subjects with only arterial hypertension as a cardiovascular indication (figure 2). We have also observed that long-term use of cardioselective $\beta$-blockers was significantly associated with a reduction in the risk of COPD exacerbations (HR 0.69, 95\% CI 0.57-0.85; $\mathrm{p}=0.001$ ) in COPD patients with a cardiovascular indication for the use of $\beta$-blockers (data not shown).

Furthermore, current use of non-cardioselective $\beta$-blockers was significantly associated with an increased risk of COPD exacerbations (HR 2.91, 95\% CI 1.65-5.13) in patients with only hypertension as cardiovascular indication, but the numbers were low (figure 2).

\section{Discussion}

In this prospective cohort study, we found that current use of cardioselective $\beta$-blockers was associated with a reduced risk of COPD exacerbations in patients with a cardiovascular indication for $\beta$-blockers use. The sensitivity analysis also indicated that the reduced risk associated with the current use of cardioselective $\beta$-blockers in COPD patients with a cardiovascular indication for $\beta$-blockers was almost similar in patients with only hypertension as a cardiovascular indication versus patients with ischaemic heart disease, atrial fibrillation and/or heart failure as a cardiovascular indication.

The use of $\beta$-blockers is frequently withheld in patients with COPD and concurrent cardiovascular disease due to concerns about potential adverse pulmonary effects such as bronchospasm. In fact, the noncardioselective $\beta$-blocker, such as propranolol, may deter the bronchodilator response to $\beta_{2}$-agonists in COPD patients [27]. However, clinical trials and meta-analyses have indicated that the use of cardioselective $\beta$-blockers did not have a significant effect on $\mathrm{FEV}_{1}$, response to $\beta_{2}$-agonists or respiratory symptoms in COPD patients $[14,28,29]$. The results of a murine model indicated that chronic use of $\beta$-blockers could reduce airway inflammation and mucus production [30]. Furthermore, some cardioselective $\beta$-blockers (e.g. nebivolol), might modify nitric oxide production, resulting in vasodilation and cardioprotective activity in hypertensive subjects with COPD [31, 32]. Additionally, $\beta$-blockers have been reported to reduce the release and synthesis of endothelin-1, a bronchoconstrictor peptide that mediated airway inflammation and may be involved in COPD exacerbations [33-35]. Due to the cardioprotective effects of $\beta$-blockers, the use of such drugs may reduce the risk of COPD exacerbations triggered by cardiovascular causes. $\beta$-blockers may reduce heart rate [36], relieve arrhythmias which can 
Strict cardiovascular indication for the use of $\beta$-blockers $(n=233)$

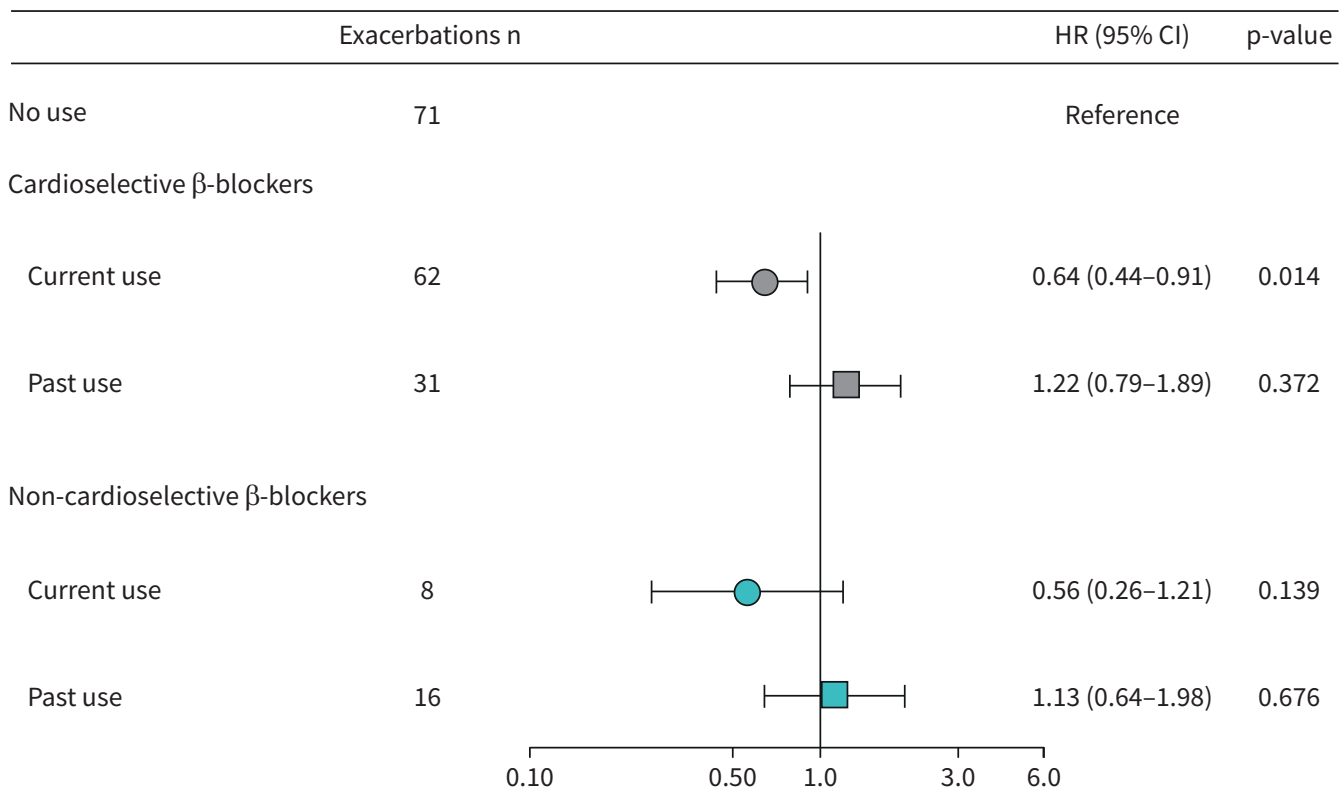

Only hypertension as indication for the use of $\beta$-blockers $(n=522)$

\begin{tabular}{|c|c|c|c|c|}
\hline & Exacerbations $\mathrm{n}$ & & $\mathrm{HR}(95 \% \mathrm{Cl})$ & $\mathrm{p}$-value \\
\hline No use & 260 & & Reference & \\
\hline \multicolumn{5}{|c|}{ Cardioselective $\beta$-blockers } \\
\hline Current use & 81 & $10-1$ & $0.69(0.54-0.89)$ & 0.005 \\
\hline Past use & 43 & & $0.99(0.71-1.38)$ & 0.969 \\
\hline \multicolumn{5}{|c|}{ Non-cardioselective $\beta$-blockers } \\
\hline Current use & 15 & & $\longmapsto 2.91(1.65-5.13)$ & 0.0002 \\
\hline Past use & 10 & $\square \longrightarrow$ & $0.46(0.24-0.89)$ & 0.020 \\
\hline & 0.1 & 0.50 & 6.0 & \\
\hline
\end{tabular}

FIGURE 2 Use of $\beta$-blockers and risk of COPD exacerbations (sensitivity analyses). Strict cardiovascular indication was defined as a history of coronary heart disease, atrial fibrillation, and/or heart failure (with or without hypertension) at baseline. The analyses were adjusted for age, sex, smoking, incident cardiovascular disease (i.e., heart failure, hypertension, atrial fibrillation, and/or coronary heart disease) during follow-up, use of respiratory drugs (R03), and nitrates (C01DA). HR: hazard ratio.

lead to cardiac and respiratory decompensation [37], and moderate the risk of acute coronary syndromes associated with the use of $\beta$-agonists [38].

A previous study by RUTTEN et al. [11] reported that the use of $\beta$-blockers reduced the risk of exacerbations in patients with COPD. This association remained in patients with COPD but without overt cardiovascular disease (i.e. only hypertension as the main indication for the prescription of $\beta$-blockers). Also, Au et al. [10] assessed the association between the type of antihypertensive medication and all-cause mortality as well as COPD exacerbations among patients with COPD and concomitant hypertension, in particular receiving single-agent antihypertensive therapy. They found a significant reduction in the risk of 
mortality associated with the $\beta$-blockers use, compared to calcium channel blockers and all other antihypertensive agents, among COPD patients with hypertension and cardiac disease, but not in the category of COPD patients with hypertension and without cardiac disease. However, the association between the use of $\beta$-blockers and the risk COPD exacerbations was not statistically significant [10]. In the current study, we add to the literature by investigating the effect of $\beta$-blockers on the risk of COPD exacerbations in COPD patients, stratified by the presence of cardiovascular conditions (defined as a history of hypertension, coronary heart disease, atrial fibrillation and/or heart failure) or absence of any cardiovascular conditions at baseline. Furthermore, we also adjusted for cardiovascular disease occurring during follow-up. A meta-analysis of 15 observational cohort studies confirmed that the use of $\beta$-blockers in patients with COPD might decrease the risk of overall mortality and reduce the risk of COPD exacerbations [13]. Furthermore, the results of a large multicentre cohort study (COPDGene) indicated that $\beta$-blockers are associated with a significant reduction of the risk of COPD exacerbations regardless of the severity of airflow obstruction [8]. Although observational studies using real-world data play an essential role in providing evidence on the effects of medications, they could be subject to methodological limitations, such as residual confounding [31]. Therefore, randomised clinical trials are essential to enhance the knowledge about the potential beneficial effects of the use of $\beta$-blockers in COPD patients.

Recently, Dransfield et al. [19], in the BLOCK COPD trial, assigned COPD patients randomly to metoprolol or placebo, with risk of first COPD exacerbation as primary endpoint; however, they excluded COPD patients with an obvious cardiovascular disease, and thus, enrolled only COPD patients without an indication for treatment with a $\beta$-blocker. Although the $\mathrm{FEV}_{1}$ was similar in the two groups, there was a higher risk of severe exacerbation (hospitalisation) and very severe exacerbation (intubation and mechanical ventilation) among the patients who received metoprolol. Furthermore, there was no significant difference in the median time until the first exacerbation in the metoprolol group versus the placebo group [19]. Our results indicated that in subjects without a cardiovascular indication, the use of cardioselective $\beta$-blockers did not significantly alter the risk of COPD exacerbations; however, due to the limited sample size of $\beta$-blockers users in this category, we might be underpowered to find a significant association.

We found that in patients with a cardiovascular indication for the use of $\beta$-blockers, current use of cardioselective $\beta$-blockers was associated with a reduced risk of COPD exacerbations. Our study reconciles the results of previous observational studies $[8,12,13]$ with the results of the BLOCK COPD RCT [19]. Previous observational studies included COPD patients regardless of concomitant cardiovascular disease, whereas the BLOCK COPD RCT excluded COPD patients with an established indication for the use of $\beta$-blockers. Therefore, the findings of the BLOCK COPD trial are relevant only to COPD patients without an indication for the use of $\beta$-blockers.

As for all observational research, our study has strengths and limitations. An important strength of our study is the fact that we used data from the Rotterdam Study which is an ongoing prospective population-based cohort with an prolonged follow-up of more than 20 years. Data were prospectively collected for all participants, independent of research questions or forthcoming diseases, which makes it less prone to information and selection bias. Furthermore, in this study we used a cohort with complete coverage of all filled prescriptions. We also analysed exposures as time-dependent variables in a Cox regression model [25].

A potential limitation of our study is that spirometry data were only available after 2002. Hence it could lead to an underestimation of asymptomatic COPD in the Rotterdam Study before 2002. In addition, the use of pre-bronchodilator spirometry implies the possibility of misclassification of some asthma patients as COPD patients in the Rotterdam Study. To control this limitation, we additionally identified and validated patients with physician-diagnosed asthma and excluded them (figure 1). In the Rotterdam Study, COPD exacerbations (moderate and severe) were recorded based on pharmacy-filled prescription data and a national hospitalisation register, which prevents recall bias compared to self-reporting of the COPD exacerbations [22]. However, there is the potential of misdiagnosis of COPD exacerbations (especially in patients with heart failure) as differentiating between worsening of heart failure symptoms and COPD exacerbations is not easy in daily life [39]. Also, for prevalent COPD, we did not have information on COPD exacerbations prior to the start of the Rotterdam Study and thus could not account for this in the analysis. Besides, lung function data at baseline were not available for all COPD patients; therefore, we were not able to consider the severity of COPD at baseline in the analysis. Moreover, smoking status was assessed at four-yearly intervals through questionnaires explaining why we categorised smoking into ever and never-smoking. Also, the use of $\beta$-blockers was based on pharmacy dispensing data and not on actual intake which might result in an overestimation of actual use. Furthermore, 23\% of patients without a cardiovascular indication for the use of $\beta$-blockers at baseline later developed incident cardiovascular disease which was controlled for in the analysis. Moreover, we acknowledge that the limited sample size of 
$\beta$-blockers users in the category without a cardiovascular indication is the main limitation of our study. Finally, although we adjusted for potential confounding factors, residual confounding might remain.

In conclusion, we observed that the use of cardioselective $\beta$-blockers decreased the risk of exacerbations in COPD patients with a cardiovascular indication for $\beta$-blockers use. Therefore, the potential benefits of $\beta$-blockers might be confined to COPD patients with cardiovascular disease.

Conflict of interest: L. Karimi has nothing to disclose. L. Lahousse reports grants from AstraZeneca and Chiesi (both awards), and expert consultation for Boehringer Ingelheim GmbH and Novartis, outside the submitted work. P. De Nocker has nothing to disclose. B.H. Stricker has nothing to disclose. G.G. Brusselle reports personal fees from AstraZeneca, Boehringer Ingelheim, Chiesi, Novartis, GlaxoSmithKline, Sanofi and Teva, outside the submitted work. K.M.C. Verhamme works for a research group that, in the past, has received unconditional research grants from Pfizer, Boehringer Ingelheim, Yamanouchi and GSK, none of which is related to the content of this paper.

Support statement: The Rotterdam Study is funded by Erasmus MC and Erasmus University, Rotterdam, the Netherlands; the Netherlands Organization for Scientific Research (NWO); the Netherlands Organization for Health Research and Development (ZonMw); the Research Institute for Diseases in the Elderly (RIDE); the Ministry of Education, Culture and Science; the Ministry for Health, Welfare and Sports; the European Commission (DG XII); and the Municipality of Rotterdam.

\section{References}

1 Singh D, Agusti A, Anzueto A, et al. Global strategy for the diagnosis, management, and prevention of chronic obstructive lung disease: The GOLD science committee report 2019. Eur Respir J 2019; 53: 1900164.

2 GOLD. Global Strategy for Diagnosis and Prevention of COPD. 2020. https://goldcopd.org/gold-reports/ Date last accessed: August 1, 2020.

3 Anees Ur R, Ahmad Hassali MA, Muhammad SA, et al. The economic burden of chronic obstructive pulmonary disease (COPD) in the USA, Europe, and Asia: Results from a systematic review of the literature. Expert Rev Pharmacoeconom Outcomes Res 2020; 20: 661-672.

4 Chen W, Thomas J, Sadatsafavi M, et al. Risk of cardiovascular comorbidity in patients with chronic obstructive pulmonary disease: A systematic review and meta-analysis. Lancet Respir Med 2015; 3: 631-639.

5 Dezsi CA, Szentes V. The real role of beta-blockers in daily cardiovascular therapy. Am J Cardiovascular Drugs: Drugs, Devices Other Intervent 2017; 17: 361-373.

6 Williams B, Mancia G, Spiering W, et al. 2018 ESC/ESH Guidelines for the management of arterial hypertension. Eur Heart J 2018; 39: 3021-3104.

7 Yancy CW, Jessup M, Bozkurt B, et al. 2017 ACC/AHA/HFSA Focused Update of the 2013 ACCF/AHA Guideline for the Management of Heart Failure: A Report of the American College of Cardiology/American Heart Association Task Force on Clinical Practice Guidelines and the Heart Failure Society of America. Circulation 2017; 136: e137-e161.

8 Bhatt SP, Wells JM, Kinney GL, et al. Beta-blockers are associated with a reduction in COPD exacerbations. Thorax 2016; 71: 8-14.

9 Short PM, Lipworth SI, Elder DH, et al. Effect of beta blockers in treatment of chronic obstructive pulmonary disease: a retrospective cohort study. BMJ 2011; 342: d2549.

$10 \mathrm{Au}$ DH, Bryson CL, Fan VS, et al. Beta-blockers as single-agent therapy for hypertension and the risk of mortality among patients with chronic obstructive pulmonary disease. Am J Med 2004; 117: 925-931.

11 Rutten FH, Zuithoff NP, Hak E, et al. Beta-blockers may reduce mortality and risk of exacerbations in patients with chronic obstructive pulmonary disease. Arch Intern Med 2010; 170: 880-887.

12 Etminan M, Jafari S, Carleton B, et al. Beta-blocker use and COPD mortality: a systematic review and meta-analysis. BMC Pulm Med 2012; 12: 48.

13 Du Q, Sun Y, Ding N, et al. Beta-blockers reduced the risk of mortality and exacerbation in patients with COPD A meta-analysis of observational studies. PloS one 2014; 9: e113048.

14 Salpeter S, Ormiston T, Salpeter E. Cardioselective beta-blockers for chronic obstructive pulmonary disease. Cochrane Database Systemat Rev 2005; 4: CD003566.

15 Dransfield MT, Rowe SM, Johnson JE, et al. Use of beta blockers and the risk of death in hospitalised patients with acute exacerbations of COPD. Thorax 2008; 63: 301-305.

16 Lipworth B, Skinner D, Devereux G, et al. Underuse of beta-blockers in heart failure and chronic obstructive pulmonary disease. Heart (Br Cardiac Soc) 2016; 102: 1909-1914.

17 Lim KP, Loughrey S, Musk M, et al. Beta-blocker under-use in COPD patients. Int J Chron Obstruct Pulmon Dis 2017; 12: 3041-3046.

18 Egred M, Shaw S, Mohammad B, et al. Under-use of beta-blockers in patients with ischaemic heart disease and concomitant chronic obstructive pulmonary disease. QJM: Monthly J Assoc Phys 2005; 98: 493-497.

19 Dransfield MT, Voelker H, Bhatt SP, et al. Metoprolol for the prevention of acute exacerbations of COPD. $N$ Engl J Med 2019; 381: 2304-2314.

20 Ikram MA, Brusselle G, Ghanbari M, et al. Objectives, design and main findings until 2020 from the Rotterdam Study. Eur J Epidemiol 2020; 35: 483-517.

21 Terzikhan N, Verhamme KM, Hofman A, et al. Prevalence and incidence of COPD in smokers and non-smokers: The Rotterdam Study. Eur J Epidemiol 2016; 31: 785-792.

22 Lahousse L, Seys LJM, Joos GF, et al. Epidemiology and impact of chronic bronchitis in chronic obstructive pulmonary disease. Eur Respir J 2017; 50: 1602470.

23 Karimi L, Lahousse L, Ghanbari M, et al. Beta2-adrenergic receptor (ADRB2) gene polymorphisms and risk of COPD exacerbations: The Rotterdam study. J Clin Med 2019; 8: 1835.

24 Leening MJ, Kavousi M, Heeringa J, et al. Methods of data collection and definitions of cardiac outcomes in the Rotterdam Study. Eur J Epidemiol 2012; 27: 173-185. 
Stricker BH, Stijnen T. Analysis of individual drug use as a time-varying determinant of exposure in prospective population-based cohort studies. Eur J Epidemiol 2010; 25: 245-251.

Finks SW, Rumbak MJ, Self TH. Treating hypertension in chronic obstructive pulmonary disease. $N$ Engl J Med 2020; 382: 353-363.

Chang CL, Mills GD, McLachlan JD, et al. Cardio-selective and non-selective beta-blockers in chronic obstructive pulmonary disease: Effects on bronchodilator response and exercise. Intern Med J 2010; 40: 193-200.

Albouaini K, Andron M, Alahmar A, et al. Beta-blockers use in patients with chronic obstructive pulmonary disease and concomitant cardiovascular conditions. Int J Chron Obstruct Pulmon Dis 2007; 2: 535-540.

Kargin F, Takir HB, Salturk C, et al. The safety of beta-blocker use in chronic obstructive pulmonary disease patients with respiratory failure in the intensive care unit. Multidiscip Respir Med 2014; 9: 8.

Nguyen LP, Omoluabi O, Parra S, et al. Chronic exposure to beta-blockers attenuates inflammation and mucin content in a murine asthma model. Am J Respir Cell Mol Biol 2008; 38: 256-262.

Dal Negro R. Pulmonary effects of nebivolol. Ther Adv Cardiovasc Dis 2009; 3: 329-334.

Kamp O, Metra M, Bugatti S, et al. Nebivolol: haemodynamic effects and clinical significance of combined beta-blockade and nitric oxide release. Drugs 2010; 70: 41-56.

Garlichs CD, Zhang H, Mügge A, et al. Beta-blockers reduce the release and synthesis of endothelin-1 in human endothelial cells. Eur J Clin Invest 1999; 29: 12-16.

Dunzendorfer S, Wiedermann CJ. Modulation of neutrophil migration and superoxide anion release by metoprolol. J Mol Cell Cardiol 2000; 32: 915-924.

5 Roland M, Bhowmik A, Sapsford RJ, et al. Sputum and plasma endothelin-1 levels in exacerbations of chronic obstructive pulmonary disease. Thorax 2001; 56: 30-35.

6 Nodari S, Metra M, Dei Cas L. Beta-blocker treatment of patients with diastolic heart failure and arterial hypertension. A prospective, randomised, comparison of the long-term effects of atenolol vs. nebivolol. Eur J Heart Fail 2003; 5: 621-627.

7 Bhatt SP, Nanda S, Kintzer JS. Arrhythmias as trigger for acute exacerbations of chronic obstructive pulmonary disease. Respir Med 2012; 106: 1134-1138.

$\mathrm{Au} \mathrm{DH}$, Curtis JR, Every NR, et al. Association between inhaled beta-agonists and the risk of unstable angina and myocardial infarction. Chest 2002; 121: 846-851.

Hawkins NM, Petrie MC, Jhund PS, et al. Heart failure and chronic obstructive pulmonary disease: Diagnostic pitfalls and epidemiology. Eur J Heart Fail 2009; 11: 130-139.

Ligthart S, van Herpt TT, Leening MJ, et al. Lifetime risk of developing impaired glucose metabolism and eventual progression from prediabetes to type 2 diabetes: A prospective cohort study. Lancet Diabetes Endocrinol 2016; 4 $44-51$. 